\title{
Original Paper \\ Dunes in the North coast of Sergipe, Brazil: plant species and their ecological traits
}

\author{
Eduardo Vinícius da Silva Oliveira ${ }^{1,2,3}$ \& Myrna Friederichs Landim ${ }^{1}$
}

\begin{abstract}
Restinga vegetation, which is a vegetation type of coastal zones, are present in two geomorphological features in the North coast of Sergipe state - Brazil: litoraneous dunes (LD) and continental dunes (CD). The objective of the present paper is to record the species composition, Raunkiaer's life forms, dispersal syndromes and soil fertility differences between both habitats. Furthermore, we compared the life forms spectra of the studied features to the spectra of the main Brazilian phytogeographic domains. We conducted a floristic survey from November 2013 to May 2014 in different Restinga areas in two dune habitats in coastal Sergipe state, Brazil, and compared differences in the two localities. Although the two areas were geographically close, there was low floristic similarity between the two habitats; CD showed higher richness than LD. The spectrum of life forms was more similar to that of Caatinga, probably due to environmental similarities. Life forms and dispersal syndromes proportions significantly differed between features, as well as the $\mathrm{pH}$, organic matter, phosphorus, sodium and base saturation index. Zoochory prevailed in both features, whereas phanerophytes prevailed in CD and chamaephytes and phanerophytes in LD. Despite the influence of historical processes, micro-environmental differences between features, which were amplified by their different distances from the sea, were assumingly responsible for the recorded differences.
\end{abstract}

Key words: dispersal syndromes, floristic similarity, Raunkiaer's life forms, Restingas.

\section{Resumo}

No litoral norte de Sergipe, a vegetação de Restinga ocorre sobre duas feições geomorfológicas: dunas litorâneas (DL) e continentais (DC). O objetivo do presente trabalho é registrar as diferenças na composição de espécies, formas de vida de Raunkiaer, síndromes de dispersão e fertilidade do solo entre ambos os habitats. Adicionalmente, comparamos os espectros de formas de vida das feições com aqueles espectros dos principais domínios fitogeográficos brasileiros. Conduzimos um levantamento florístico durante o período de novembro/2013 a maio/2014 nas duas feições geomorfológicas analisadas. Embora as duas áreas sejam geograficamente próximas, houve baixa similaridade florística entre os dois habitats, tendo DC apresentado maior riqueza de espécies do que DL. O espectro das formas de vida foi mais similar ao da Caatinga, provavelmente em virtude de semelhanças ambientais. As proporções de formas de vida e síndromes de dispersão diferiram significativamente entre as feições, assim como os parâmetros pH, matéria orgânica, fósforo, sódio e índice de saturação por bases. A zoocooria predominou nas duas feições, enquanto que os fanerófitos predominaram em DC e caméfitos e fanerófitos, em DL. Apesar da influência de processos históricos, diferenças micro-ambientais entre as feições dunares estudadas, amplificadas pelas suas diferentes distâncias do mar, devem ser responsáveis pelas diferenças observadas.

Palavras-chave: síndromes de dispersão, similaridade florística, formas de vida de Raunkiaer, Restingas.

\footnotetext{
${ }^{1}$ Universidade Federal de Sergipe, Centro de Ciências Biológicas e da Saúde, Depto. Biologia, Cidade Universitária Prof. José Aloísio de Campos, Av. Marechal Rondom s/n, Jardim Rosa Elze, 49100-000, São Cristóvão, SE, Brazil.

${ }^{2}$ ORCID: <https://orcid.org/0000-0003-4858-3930>

${ }^{3}$ Author for correspondence: eduardovso@yahoo.com.br
} 


\section{Introduction}

Coastal dunes are natural features formed by the transportation of fine-grained beach sand by the wind (Villwock et al. 2005; Cordazzo et al. 2006; Guerra \& Guerra 2008; Guerra \& Cunha 2009). These features play a fundamental role in maintaining and preserving the integrity of the coast, since they work as dynamic barriers against the action of waves and storms (Cordazzo \& Seeeliger 1995 ) and as habitat for numerous plant and animal species (Cerqueira 2000).

The strong winds, high salinity, low soil moisture and low organic matter levels in these habitats are the most constraint factors for plant growth and development (Andrade 1967; Santos et al. 2000). Vegetation presents morphological adaptations in response to these factors, such as salt accumulation in the leaves and the development of special stems (Joly 1970; Rizzini 1997). Furthermore, many perennial plants in the coastal dunes are succulent and/or sclerophyllous (Martin et al. 1992).

Different habitats for plants result from a variety of topographic features associated with different environmental gradients in coastal dunes (Cordazzo et al. 2006). Therefore, dune vegetation is not homogeneous, but rather disposed in bands or patches, whose zonation is responsible for differences in vegetation richness and structure (Waechter 1985; Cordazzo \& Seeliger 1995; Falkenberg 1999; Cordazzo et al. 2006; AlmeidaJr. et al. 2016).

These micro-environmental variations are also responsible for vegetation diversity (Brito et al. 1993; Leite \& Andrade 2004; Cordazzo et al. 2006). Chamaephytes and geophytes, for instance, often prevail in dunes located closest to the sea, while phanerophytes are the predominant vegetation in dunes more distant to the water (Guara et al. 1992; Martin et al. 1992; Henriques \& Carlos-Neto 2002). Greater relative importance of anemochory has been observed in the windward of dunes (Maun 2009) and of zoochory to leeward (Marques \& Oliveira 2005).

Coastal dunes are one of the richest Restinga formations; estimates show that they house 31 to 410 angiosperms species, belonging to 28-283 genera and to 11-88 families (Cordazzo \& Seeliger 1995; Amaral et al. 2008). Leguminosae is the family with the largest number of species in these environments, which is followed by families Asteraceae and Poaceae (in decreasing order of importance) in coastal dunes in Northern (Santos
\& Rosário 1988; Araujo et al. 2016), Northeastern (Brito et al. 1993; Santos et al. 2000, 2011; Leite \& Andrade 2004; Viana et al. 2006; Almeida-Jr. et al. 2016), Southeastern (Castelo \& Braga 2017) and Southern Brazil (Palma \& Jarenkow 2008; Ribeiro \& Melo-Jr. 2016).

Besides the dunes in the Coastal Plains, that compose the geomorphological feature of litoraneous dunes (dunas litorâneas) in the North coast of Sergipe State, dunes are also found on the Coastal Tablelands (Tabuleiros Costeiros), which are geomorphological units associated with Barreiras Formation sediments, represent the features of the so-called continental dunes (dunas continentais) (Fontes 1985; Guerra \& Guerra 2008; Alves 2010). Quaternary sand dunes or sandy bands over tertiary deposits in the Coastal Tablelands are present in other Northeastern Brazil coastal regions such as Ceará (Moro et al. 2015), Rio Grande do Norte (Freire 1990; Almeida-Jr. et al. 2006), Paraíba (Oliveira-Filho \& Carvalho 1993) and Bahia states (Rizzini 1997).

In the Restingas of Sergipe, 831 species of angiosperms have been recorded (Oliveira et al. 2014), being Leguminosae the family with the highest specific richness (Oliveira et al. 2014, 2015; Nascimento-Jr. 2012). The phytophysiognomies found in the Restingas of Sergipe are diverse and similar to other areas of this vegetation in the Northeast Brazil (Oliveira \& Landim 2014), presenting rare and endangered species (Oliveira \& Landim 2016) despite the intense anthropic degradation (Pergentino \& Landim 2014). Floristic and/or taxonomic studies were carried out in the Restingas of the Biological Reserve (REBIO) of Santa Isabel (Oliveira et al. 2015), in the Santo Amaro das Brotas County (Nascimento-Jr. 2012), and in the Coastal Tablelands of the Pirambu County (Silva 2014), all of these located in the north coast of the state.

Despite the scientific importance of and conservation risk faced by dune ecosystems, given the intense anthropic pressure on them (Santos \& Rosário 1988; Santos et al. 2000; Leite \& Andrade 2004; Melo-Jr. et al. 2017), publications on their flora in Sergipe State remain scarce; unlike observed for Restinga areas in general, where studies have increased recently. Accordingly, the aim of the present paper is to compare the flora of litoraneous and continental dunes in order to assess the composition, richness, frequency of Raunkiaer's life forms and dispersal syndromes of plant species and the soil fertility of two geomorphological dune 
features in the Northern coast of Sergipe state. Furthermore, we compared the life forms spectra of the studied features to the spectra of the main Brazilian phytogeographic domains.

\section{Material and Methods}

Study site

We conducted the study in two different areas, one inside and another in the vicinity, of the Biological Reserve (REBIO) of Santa Isabel, Pirambu County, North coast of Sergipe state, Northeastern Brazil (Figs. 1, 2). According to its creation decree, Santa Isabel Biological Reserve, which is located between Pirambu and Pacatuba Counties, covers 2,766 ha and comprises a $45 \mathrm{~km}$ long coastline (Brasil 1988).

Based on the Köppen's climate classification system, the region is of the As type, (Alvares et al. 2013). It records mean annual temperature of $26{ }^{\circ} \mathrm{C}$, and the mean annual rainfall is $1,650 \mathrm{~mm}$; and the rainy season goes from March to August (SEPLAG-SE 2011). The Quartzarenic Neosol soil type prevails in the assessed REBIO, which is acidic and presents high permeability and low fertility (EMBRAPA 2006; Fraga 2010). The soil types Podzolic and Halomorphic are also found throughout this REBIO's, as well as in its surrounding areas (EMBRAPA/SUDENE 1975).

Santa Isabel Biological Reserve is predominantly covered by Restinga vegetation. It presents seven phytophysiognomic types, like graminous fields, as well as coastal scrubs, flooded and non-flooded forests (Oliveira \& Landim 2014). Oliveira et al. (2015) recorded 260 species distributed in 184 genera and 78 families in the area.

According to the limits of each geomorphological habitat (Alves 2010; SEMARH/ SRH-SE 2013), we initially assessed the dunes selected for sampling in the REBIO by analyzing their aerial photographs (SEPLANTEC-SE 2003). We selected four sampling sites (Figs. 1, 2 ); three of them were on the Coastal Plain, in the litoraneous dune (LD) feature, which were located approximately $100 \mathrm{~m}$ from the coastline. We also chose a more extensive sampling area of the continental dune (CD) feature located in Coastal Tablelands, approximately $2 \mathrm{~km}$ from the coastline. This area was chosen because it holds the only continental dune in the REBIO limits according to a proposal to extend the limits of the REBIO (Santos et al. 2017).
We obtained the geographical coordinates of each sampling site in the field using a GPS (collected in field), and delimited the limits of each dune and their total area using a GIS software. The continental dune is approximately $11,500 \mathrm{~m}^{2}$, and this area is equivalent to the three litoraneous dunes sampled, which, together, cover an area of approximately $11,600 \mathrm{~m}^{2}$.

\section{Data collection}

We conducted a floristic sampling through opportunistic collection of plant material during hiking from October 2013 to May 2014 (Filgueiras et al. 1994) in the two studied dune fields in order to search for material, mainly at its reproductive stage. Eight field samplings were carried out in each of the two studied features (sixteen in all), being two campaigns per month. Each field sampling lasted six hours, on average. All four dunes were sampled in every field LD campaign. We deposited the collected material in the ASE Herbarium of Federal University of Sergipe and herborized according to usual methods (Mori et al. 1989).

The collected material was identified based on the specialized literature (Souza \& Lorenzi 2012; Prata et al. 2013, 2015; Martins-da-Silva et al. 2014) and on comparisons to the material registered at the ASE Herbarium. We used the floristic survey applied to Santa Isabel Biological Reserve to help identifying non-fertile specimens (Oliveira et al. 2015). Classification of species in families followed the APG IV (2016) classification system and the spelling of name of species was checked in BFG (2018).

Soil collection was based on the methodology by Silva (1999) and carried out in top dune areas in the two assessed dune features: 12 samples (nine in LD and three in CD), in total. We collected three samples in CD under different shrub-tree vegetation islands presenting the best development stage among other islands of that feature. The topsoil was removed (including the litter) and a $0.5 \mathrm{~kg}$ fraction of soil was collected from the layer $20-\mathrm{cm}$ down the surface at these sites. The same sampling methodology was adopted for each of the three sampled dunes in LD; three soil samples were collected in areas covered with plants for each of the sampled dunes.

We sent the collected material to the Laboratório de Solos e Química Agrícola do Instituto Tecnológico e de Pesquisas do Estado de Sergipe (Soils and Agricultural Chemistry Laboratory of Sergipe State Technological and 

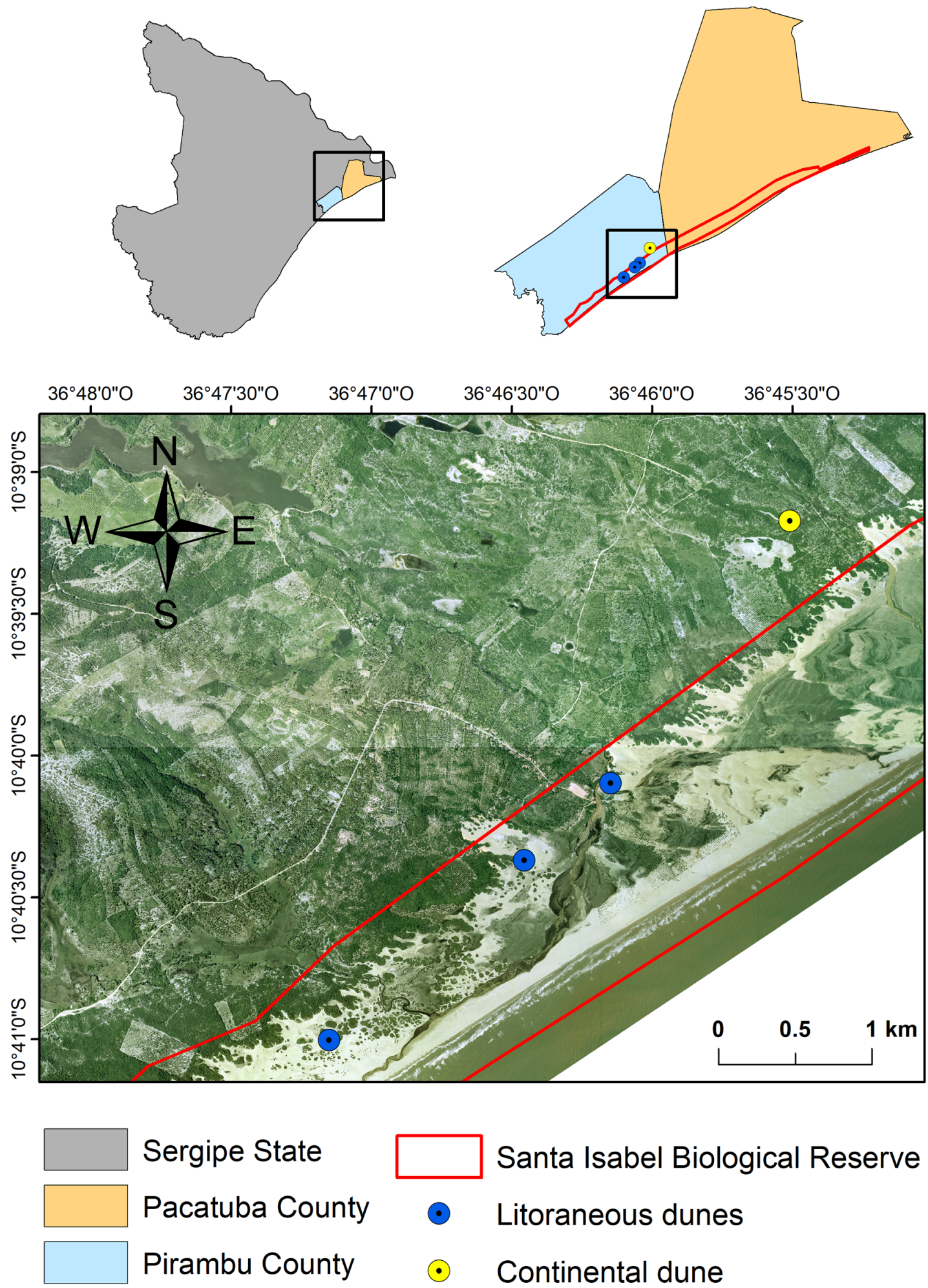

Sergipe State

\section{Pacatuba County}

Pirambu County

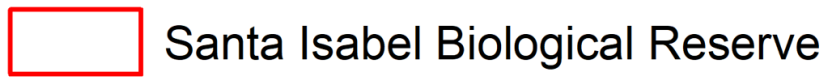

- Litoraneous dunes

$-\quad$ Continental dune

Figure 1 - Sampling points of the dune features sampled, being one inside and another in the vicinity, of the Biological Reserve (REBIO) of Santa Isabel, Pirambu County, North coast of Sergipe state, Northeastern Brazil. Boundaries of REBIO Santa Isabel according ICMBio (2017). Source of polygons and aerial photographs: Atlas Digital - SRH 2013/ SEPLANTEC-SE 2003. Preparation: Eduardo V.S. Oliveira, UTM Projection; Datum Sirgas 2000, Zone 24 S. 
Research Insitute) in order to be subjected to fertility analysis $(\mathrm{pH}$, organic matter, aluminum, calcium, phosphorus, magnesium, potassium, sodium, exchangeable bases, cation exchange capacity and base saturation index) according to standardized methods (Silva 1999).

\section{Data analysis}

A species accumulation (Gurevitch et al. 2009) curve was generated to check the sufficiency of the sample from each feature by taking into consideration that each field campaign was a sampling unit.

Morphological characteristics such as color and size, as well as the accessory structures potentially attractive to dispersing agents were initially analyzed to classify the diaspores of dispersal syndromes. Subsequently, the morphological characterization of fruits (Barroso et al. 2004; Lorenzi et al. 2006) and the classification of their dispersal syndrome (Pijl 1982) were carried out. Dispersal syndrome classification of non-fertile specimens was done by consulting the scientific literature. Finally, the collected species were classified based on Raunkiaer's life-forms (Martins \& Batalha 2011) by using the key classification proposed by Mueller-Dombois \& Ellenberg (1974). The floristic similarity between the two dune features was calculated based on the Sørensen Index $\left(\mathrm{S}_{\mathrm{I}}\right)$ (Mueller-Dombois \& Ellenberg 1974).

The proportion of Raunkiaer's life forms of the studied features were compared to the life form spectra of the main Brazilian phytogeographic domains (Costa et al. 2016). We used the lifeforms in our sites to compare to a database with the life-form spectra of 21 areas in Ombrophilous and semideciduous Atlantic forests, Amazon Rainforest, Cerrado (Central Brazilian Savanna) and Crystalline and Sedimentary Caatinga (Seasonally Dry Forests) (Costa et al. 2016). The relation between spectra of life forms in each area was evaluated through Non-Metric Multidimensional Scaling (NMDS) by using the Euclidian distances. This analysis was performed in the vegan package of the R software (R Development Core Team 2013; Oksanen et al. 2018).
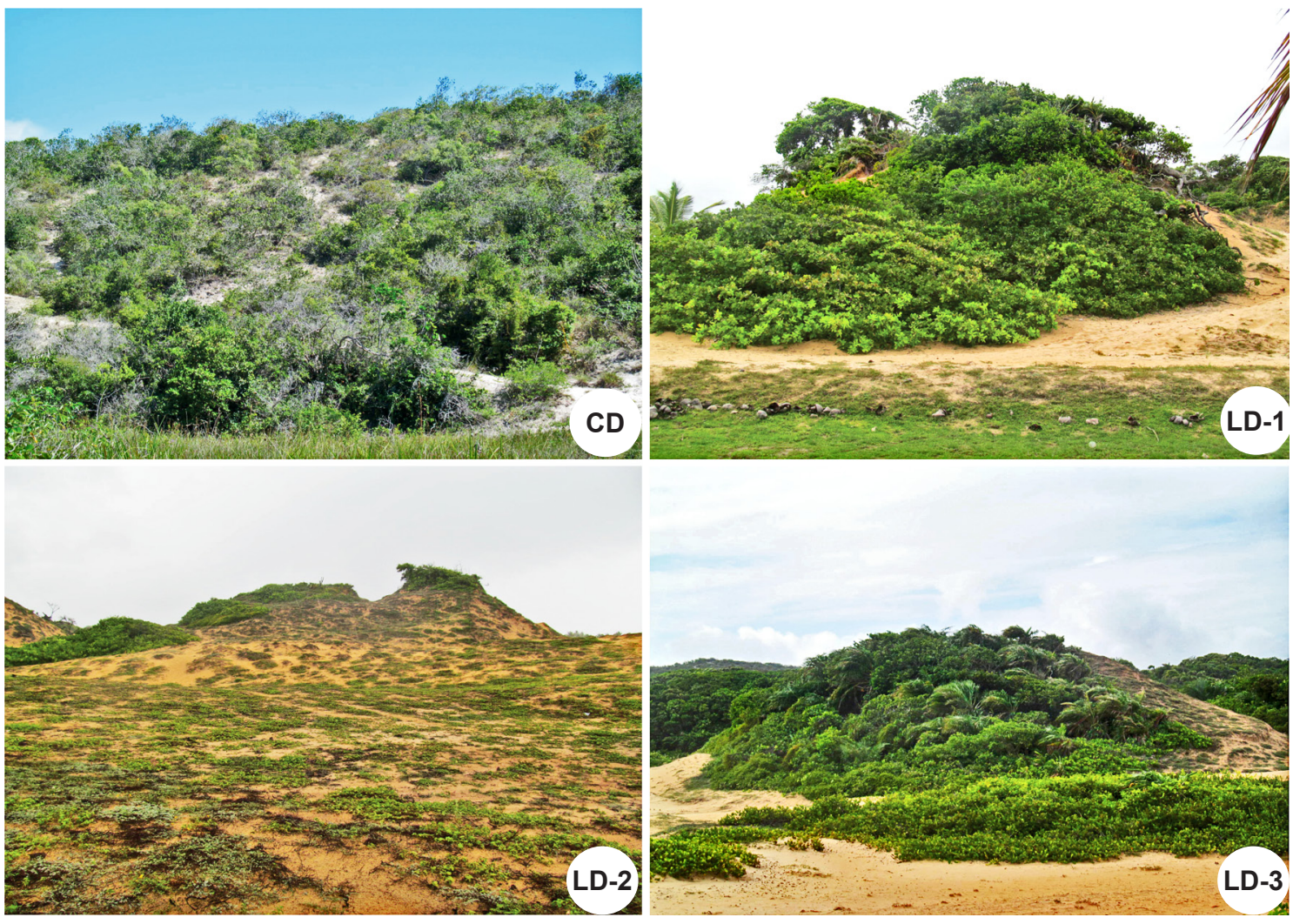

Figure 2 - Sampled dunes in the studied features of Santa Isabel Biological Reserve, Pirambu County, Northern coast of Sergipe state, Northeastern Brazil. $\mathrm{CD}=$ continental dune; $\mathrm{LD}=$ litoraneous dunes. 
The chi-square test $\left(\mathrm{x}^{2}\right)$ was applied to assess differences in the proportion of dispersal syndromes and Raunkiaer's life forms in the two analyzed features. The null hypothesis $\left(\mathrm{H}_{0}\right)$ of equality in the proportion between the analyzed factors was tested. The Mann-Whitney U-Test $\left(\alpha=0.05 ; U_{\text {critical }}=2\right)$, suitable for small samples (Siegel 1977), was used to find whether soil fertility presented significant difference between the two dune features. All statistical tests were performed using the software Past 2.17 statistical (Hammer et al. 2013).

\section{Results}

We list 91 species of angiosperms distributed in 43 families in the four dunes sampled (Tab. 1). The highest number of species and family richness was observed in CD (Tab. 2). Whereas Leguminosae and Myrtaceae (six species each), and Rubiaceae (five) were the richest families in number of species in CD (Fig. 3a), Leguminosae (six species), Poaceae (four) and Asteraceae and Rubiaceae (three species each) were the richest families in species number in LD.

Table 1 - Angiosperms collected in dunes studied in the interior and surroundings of Biological Reserve of Santa Isabel, north coast of Sergipe state, Northeastern Brazil. $\mathrm{CD}=$ continental dune feature; $\mathrm{LD}=$ litoraneous dunes feature; $\mathrm{FT}=$ fruit type; $\mathrm{Ac}=$ achene $; \mathrm{Ba}=$ bacca $\mathrm{Ca}=$ capsule $; \mathrm{Cr}=$ caryopsis $; \mathrm{Cy}=$ cypsela $; \mathrm{Dr}=$ drupe; $\mathrm{Sc}=$ schizocarp; $\mathrm{Fo}=$ follicle; $\mathrm{Le}=$ legume; $\mathrm{Lo}=$ lomentum; $\mathrm{Nu}=$ nucula (nut type); Py = pyxidium; $\mathrm{DS}=$ dispersal syndrome; An = anemochory; $\mathrm{Au}=$ autochory; $\mathrm{Zo}=$ zoochory; $\mathrm{LF}=$ Raunkiaer's life forms; $\mathrm{Ch}=$ chamaephyte; $\mathrm{Ph}=$ phanerophyte; $\mathrm{Hc}=$ hemicryptophyte; $\mathrm{He}=$ hemiepiphyte; $\mathrm{Th}=$ therophyte.

\begin{tabular}{|c|c|c|c|c|c|c|c|c|c|}
\hline Family / Species & CD & LD1 & LD2 & LD3 & FT & DS & $\mathbf{L F}$ & Collector number & $\begin{array}{c}\text { Voucher } \\
\text { (ASE register } \\
\text { number) }\end{array}$ \\
\hline \multicolumn{10}{|l|}{ Anacardiaceae } \\
\hline Anacardium occidentale L. & $\mathrm{x}$ & $\mathrm{x}$ & $\mathrm{x}$ & $\mathrm{x}$ & $\mathrm{Nu}$ & Zo & $\mathrm{Ph}$ & $\begin{array}{l}\text { E.V.S. Oliveira } 255 \text {, } \\
259\end{array}$ & 29411,29582 \\
\hline \multicolumn{10}{|l|}{ Annonaceae } \\
\hline Duguetia moricandiana Mart. & $\mathrm{x}$ & & & & $\mathrm{Ba}$ & Zo & $\mathrm{Ph}$ & E.V.S. Oliveira 244 & 29401 \\
\hline \multicolumn{10}{|l|}{ Apocynaceae } \\
\hline Calotropis procera (Aiton) Dryand.* & & & $\mathrm{x}$ & & $\mathrm{Ca}$ & An & $\mathrm{Ph}$ & E.V.S. Oliveira 100 & 29212 \\
\hline Hancornia speciosa Gomes & $\mathrm{x}$ & & $\mathrm{x}$ & & Dr & Zo & $\mathrm{Ph}$ & E.V.S. Oliveira 249 & 29405 \\
\hline $\begin{array}{l}\text { Himatanthus bracteatus } \\
\text { (A.DC.) Woodson }\end{array}$ & $\mathrm{x}$ & & & & $\mathrm{Sc}$ & $\mathrm{Au}$ & $\mathrm{Ph}$ & E.V.S. Oliveira 413 & 30872 \\
\hline $\begin{array}{l}\text { Mandevilla moricandiana } \\
\text { (A.DC.) Woodson }\end{array}$ & $\mathrm{x}$ & & & & Fo & $\mathrm{Au}$ & $\mathrm{Ph}$ & E.V.S. Oliveira 407 & 30587 \\
\hline $\begin{array}{l}\text { Mandevilla scabra (Hoffmanns. ex } \\
\text { Roem. \& Schult.) K.Schum. }\end{array}$ & $\mathrm{x}$ & & & & Fo & $\mathrm{Au}$ & $\mathrm{Ph}$ & E.V.S. Oliveira 277 & 25598 \\
\hline \multicolumn{10}{|l|}{ Araceae } \\
\hline Anthurium affine Schott & $\mathrm{x}$ & & $\mathrm{x}$ & & $\mathrm{Ba}$ & Zo & $\mathrm{Hc}$ & $\begin{array}{l}\text { E.V.S. Oliveira } 410, \\
424\end{array}$ & 30590,30860 \\
\hline Philodendron imbe Schott ex Kunth. & & $\mathrm{x}$ & & $\mathrm{x}$ & $\mathrm{Ba}$ & Zo & $\mathrm{He}$ & E.V.S. Oliveira 258 & 29581 \\
\hline \multicolumn{10}{|l|}{ Arecaceae } \\
\hline Allagoptera arenaria (Gomes) Kuntze & $\mathrm{x}$ & & & & Dr & Zo & $\mathrm{Ph}$ & E.V.S. Oliveira 414 & 30592 \\
\hline Syagrus coronata (Mart.) Becc. & & $\mathrm{x}$ & $\mathrm{x}$ & $\mathrm{x}$ & Dr & Zo & $\mathrm{Ph}$ & E.V.S. Oliveira 274 & 25595 \\
\hline \multicolumn{10}{|l|}{ Asteraceae } \\
\hline Ageratum conyzoides L. & & & $\mathrm{x}$ & & $\mathrm{Cy}$ & An & $\mathrm{Ch}$ & E.V.S. Oliveira 419 & 30857 \\
\hline $\begin{array}{l}\text { Conocliniopsis prasiifolia } \\
\text { (DC.) R.M.King \& H.Rob. }\end{array}$ & & $\mathrm{x}$ & $\mathrm{x}$ & $\mathrm{x}$ & $\mathrm{Cy}$ & An & $\mathrm{Th}$ & E.V.S. Oliveira 271 & 25594 \\
\hline
\end{tabular}


Voucher

Family / Species

CD LD1 LD2 LD3 FT DS LF Collector number (ASE register

$\begin{array}{lllllll}\text { Conyza bonariensis (L.) Cronquist } & \mathrm{x} & \mathrm{Cy} & \mathrm{An} & \mathrm{Ch} & \text { E.V.S. Oliveira } 406 & 30586 \\ \text { Elephantopus hirtiflorus DC. } & \mathrm{x} & \mathrm{x} & \mathrm{Cy} & \text { An } & \text { Th } & \text { E.V.S. Oliveira 122, 29153, 29591 } \\ & & & & & & \end{array}$

Bignoniaceae

Lundia cordata (Vell.) DC.

Bonnetiaceae

Bonnetia stricta (Nees) Nees \& Mart. $\quad$ x

$\mathrm{Ca} \quad \mathrm{Au} \quad \mathrm{Ph} \quad$ E.V.S. Oliveira $413 \quad 30872$

Bromeliaceae

Aechmea aquilega (Salisb.) Griseb.

Hohenbergia catingae Ule

Cactaceae

Melocactus violaceus Pfeiff.

Chrysobalaceae

Hirtella racemosa Lam.

Ba Zo Hc E.V.S. Oliveira A98

29138

Clusiaceae

Symphonia globulifera L.f.

Commelinaceae

Commelina erecta $\mathrm{L}$.

$\mathrm{X}$

$\mathrm{Ba}$ Zo Hc E.V.S. Oliveira 250

29406

Convolvulaceae

Evolvulus pterocaulon Moric

Cyperaceae

Cyperus crassipes Vahl

Cyperus ligularis L.

Eleocharis geniculata

(L.) Roem. \& Schult.

\begin{tabular}{|c|c|c|c|c|c|c|}
\hline $\mathrm{x}$ & & $\mathrm{Ac}$ & An & $\mathrm{Hc}$ & E.V.S. Oliveira 415 & 30853 \\
\hline $\mathrm{x}$ & $\mathrm{x}$ & Ac & An & $\mathrm{Hc}$ & E.V.S. Oliveira 285 & 29603 \\
\hline & & $\mathrm{Ac}$ & $\mathrm{Au}$ & Th & E.V.S. Oliveira 405 & 305 \\
\hline
\end{tabular}

Dilleniaceae

Curatella americana L.

Davilla flexuosa A.St.-Hil.

Tetracera breyniana Schltdl.
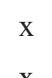

X

$\mathrm{X}$

Euphorbiaceae

Cnidosculus urens (L.) Arthur

Croton sellowii Baill

$\mathrm{X}$

Krameriaceae

Krameria tomentosa A.St.-Hil.

$\mathrm{X}$

Lamiaceae

Hypenia salzmannii (Benth.) Harley

$\mathrm{X}$

Hyptis fruticosa Salzm. ex Benth.

Marsypianthes chamaedrys (Vahl) Kuntze
$\mathrm{Nu} \quad \mathrm{Zo} \quad \mathrm{Ph}$ E.V.S. Oliveira $303 \quad 30047$

$\mathrm{Ca} \quad \mathrm{Zo} \quad \mathrm{Ph}$ E.V.S. Oliveira $291 \quad 30063$

$\mathrm{Ba} Z \mathrm{Zo} \quad \mathrm{Ph}$ E.V.S. Oliveira 301, 30051, 29589 266
29602

29393

Lauraceae 
Family / Species

CD LD1 LD2 LD3 FT DS LF Collector number (ASE register number)

Cassytha filiformis L.

$\begin{array}{lllllllll}\text { x } & \text { x } & \text { x } & \text { x } & \text { Ba } & \text { Zo } & \text { Ph } & \text { E.V.S. Oliveira 236, 29395, } 30856\end{array}$

417

Lecythidaceae

Eschweilera ovata $\quad$ x

(Cambess.) Mart. ex Miers

Lecythis pisonis Cambess.

Leguminosae

Aeschynomene viscidula Michx.

Andira fraxinifolia Benth.

Centrosema virginianun (L.) Benth.

Centrosema brasilianum (L.) Benth.

Chamaecrista flexuosa (L.) Greene

Chamaecrista hispidula

(Vahl) H.S.Irwin \& Barneby

Chamaecrista ramosa

(Vogel) H.S.Irwin \& Barneby

Stylosanthes viscosa (L.) Sw.

Lythraceae

Cuphea flava Spreng

Malpighiaceae

Byrsonima gardnerana A. Juss.

Byrsonima sericea DC.

Stigmaphyllon paralias A.Juss.

Malvaceae

Sida angustissima A.St.-Hil.

Sida spinosa L.

Melastomataceae

Comolia ovalifolia (DC.) Triana

Mouriri pusa Gardner

Myrtaceae

Calycolpus legrandii Mattos

Eugenia punicifolia (Kunth) DC.

Marlierea excoriata Mart.

Myrcia guianensis (Aubl.) DC.

Myrcia ovina Proença \& Landim

Psidium guajava L.

$\mathrm{X}$

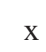

$\mathrm{X}$

X

$\mathrm{X}$

$\mathrm{X}$

$\mathrm{X}$

$$
\begin{gathered}
\mathrm{Py} \mathrm{Au} \\
\mathrm{Ph} \quad \text { E.V.S. Oliveira } \\
291 \mathrm{~A}
\end{gathered}
$$

$\mathrm{Py} \mathrm{Au} \quad \mathrm{Ph}$ E.V.S. Oliveira 412

30871

Lo Zo

Ch E.V.S. Oliveira 269

25592

Dr Zo $\mathrm{Ph}$ E.V.S. Oliveira $160 \quad 29184$

Le $\mathrm{Au}$

Th E.V.S. Oliveira 276 ,

25597, 29586

267

Le $\mathrm{Au}$ Th E.V.S. Oliveira 263

25590

Le An

Ch E.V.S. Oliveira 287, 283

Le $\mathrm{Au}$ Th E.V.S. Oliveira 247

29404

$\mathrm{X} \quad \mathrm{X} \quad \mathrm{X}$

Le $\mathrm{Au}$ Th E.V.S. Oliveira 240, 29398, 29587

264

Lo Zo Ch E.V.S. Oliveira 254, 29410, 29588 265

$\mathrm{Ca}$ An Ch E.V.S. Oliveira 251

29407

Dr Zo

$\mathrm{Ph}$

E.V.S. Oliveira 293 418 $\mathrm{X} \quad \mathrm{X} \quad \mathrm{X}$

Dr Zo

$\mathrm{Ph}$ E.V.S. Oliveira 270

29593

$\mathrm{Sc} \mathrm{Au} \mathrm{Ph}$ E.V.S. Oliveira 297

30301

$\mathrm{Sc} \mathrm{Au}$

Ch E.V.S. Oliveira 294

30065

$\mathrm{Sc} \mathrm{Au}$

Ch E.V.S. Oliveira 262

29585

$\mathrm{Ca} \mathrm{Au}$

$\mathrm{Ph}$ E.V.S. Oliveira 121

29152

$\mathrm{Ba} \mathrm{Zo}$

$\mathrm{Ph}$

E.V.S. Oliveira

30578 $299 \mathrm{~A}$

$\mathrm{Ba} \quad \mathrm{Zo} \quad \mathrm{Ph} \quad$ E.V.S. Oliveira 302

30300

$\mathrm{Ba} \quad \mathrm{Zo} \quad \mathrm{Ph}$ E.V.S. Oliveira 298

30297

$\mathrm{Ba} \quad \mathrm{Zo} \quad \mathrm{Ph}$ E.V.S. Oliveira 400

30869

$\mathrm{Ba} \quad \mathrm{Zo} \quad \mathrm{Ph} \quad$ M.F. Landim 1708

30498

$\mathrm{Ba} Z \mathrm{Zo} \quad \mathrm{Ph}$ E.V.S. Oliveira 401

30582

$\mathrm{Ba} \mathrm{Zo} \mathrm{Ph}$ E.V.S. Oliveira 403 


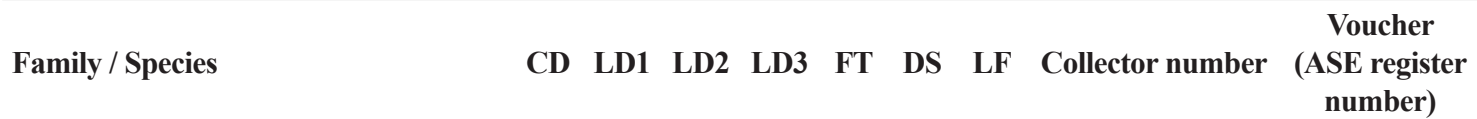

$\begin{array}{llllllll}\begin{array}{l}\text { Guapira pernambucensis } \\ \text { (Casar.) Lundell }\end{array} & \mathrm{x} & \mathrm{x} & \mathrm{Dr} & \mathrm{Zo} & \mathrm{Ph} & \begin{array}{c}\text { E.V.S. Oliveira 408, } \\ 280\end{array} & \text { 30588, 29599 } \\ \end{array}$

Ochnaceae

Ouratea cuspidata St. Hil.

Sauvagesia erecta $\mathrm{L}$.

$\mathrm{X}$

$\mathrm{X}$

Passifloraceae

Piriqueta duarteana var. duarteana (Cambess.) Urb.

Peraceae

Chaetocarpus echinocarpus

(Baill.) Ducke

Plantaginaceae

Scoparia dulcis L.

Poaceae

Andropogon selloanus (Hack.) Hack. x

Eragrostis ciliaris (L.)

Paspalum maritium Trin.

Pycreus fugax (Liebm.) C.D.Adams

Setaria tenax (Rich.) Desv.

Polygalaceae

Asemeia violacea

(Aubl.) J.F.B.Pastore \& J.R.Abbott

Polygala cyparissias

A.St.-Hil. \& Moq.

Polygonaceae

Coccoloba laevis Casar.

Portulacaceae

Portulaca halimoides L.

Rubiaceae

Borreria capitata (Ruiz \& Pav.) DC.

Borreria verticillata (L.) G.Mey.

Cordiera concolor (Cham.) Kuntze

Diodella apiculata

(Willd. ex Roem. \& Schult.) Delprete

Mitracarpus hirtus (L.) DC.

Tocoyena bullata (Vell.) Mart.

$\mathrm{X}$
Dr Zo $\quad \mathrm{Ph} \quad$ E.V.S. Oliveira 157

29181

$\mathrm{Ca} \mathrm{Au} \quad \mathrm{Ph}$ E.V.S. Oliveira $409 \quad 30589$

$\mathrm{X} \quad \mathrm{X} \quad \mathrm{X}$

$\mathrm{Ca} \mathrm{Au}$ Ch E.V.S. Oliveira 237, 29396, 30293 $267 A$

Ca Zo Ph E.V.S. Oliveira 296

30302

x $\quad \mathrm{Ca} \mathrm{Au}$ Ch E.V.S. Oliveira 286 30294

X

Cr An Hc E.V.S. Oliveira 241 29399

$\mathrm{Cr}$ An Th E.V.S. Oliveira $282 \quad 30304$

$\mathrm{x} \quad \mathrm{x} \quad \mathrm{x} \quad \mathrm{Cr}$ An Hc E.V.S. Oliveira 218, 29449, 30861

421

$\mathrm{X}$

$\mathrm{Cr}$ An Hc E.V.S. Oliveira

30357

$274 A$

$\mathrm{x} \quad \mathrm{Cr}$ An Hc E.V.S. Oliveira 404, 30584, 29600 281

x $\quad \mathrm{x} \quad \mathrm{Ca}$ An Ch E.V.S. Oliveira 243, 30352, 30295 279

Ca An Th E.V.S. Oliveira 290 30053

Dr Zo Ph E.V.S. Oliveira $426 \quad 30865$

$\mathrm{x} \quad \mathrm{x} \quad \mathrm{Py}$ Au Hc E.V.S. Oliveira 222 29413

$\mathrm{Ca} \mathrm{Au} \quad \mathrm{Ch}$ E.V.S. Oliveira 245, 29402, 29583 260

$\mathrm{Ca} \mathrm{Au}$ Ch E.V.S. Oliveira 235, 29394, 29583 260

$\mathrm{Ba} \quad \mathrm{Zo} \quad \mathrm{Ph} \quad$ E.V.S. Oliveira $278 \quad 30868$

$\mathrm{Sc} \mathrm{Au}$ Ch E.V.S. Oliveira $246 \quad 29403$

$\begin{array}{llllll}\mathrm{x} & \mathrm{x} & \mathrm{Ca} & \mathrm{Au} & \mathrm{Ch} & \text { E.V.S. Oliveira 261 } 29584\end{array}$

$\mathrm{x} \quad \mathrm{Ba} \quad \mathrm{Zo} \quad \mathrm{Ph} \quad$ E.V.S. Oliveira 427, 30864, 30858

423

Santalaceae 


\begin{tabular}{|c|c|c|c|c|c|c|c|c|c|}
\hline Family / Species & CD & LD1 & LD2 & LD3 & FT & DS & $\mathbf{L F}$ & Collector number & $\begin{array}{l}\text { Voucher } \\
\text { (ASE register } \\
\text { number) }\end{array}$ \\
\hline Phoradendron strongyloclados Eichler & $\mathrm{x}$ & & & & $\mathrm{Ba}$ & Zo & $\mathrm{Ph}$ & E.V.S. Oliveira 130 & 29161 \\
\hline \multicolumn{10}{|l|}{ Sapotaceae } \\
\hline Manilkara rufula (Miq.) H.J.Lam & $\mathrm{x}$ & & & & $\mathrm{Ba}$ & Zo & $\mathrm{Ph}$ & E.V.S. Oliveira 158 & 29182 \\
\hline \multicolumn{10}{|l|}{ Simaroubaceae } \\
\hline Simaba floribunda A.St.-Hil. & $\mathrm{x}$ & & & & Dr & Zo & $\mathrm{Ph}$ & E.V.S. Oliveira 252 & 29408 \\
\hline \multicolumn{10}{|l|}{ Verbenaceae } \\
\hline Lantana camara $\mathrm{L}$. & $\mathrm{x}$ & & & & $\mathrm{Ba}$ & Zo & $\mathrm{Ph}$ & E.V.S. Oliveira 134 & 29164 \\
\hline Lippia alba (Mill.) N.E.Br. & & & $\mathrm{x}$ & & $\mathrm{Ca}$ & $\mathrm{Au}$ & $\mathrm{Ch}$ & E.V.S. Oliveira 422 & 30873 \\
\hline Stachytarpheta microphylla Walp. & $\mathrm{x}$ & & & & $\mathrm{Sc}$ & $\mathrm{Au}$ & $\mathrm{Ch}$ & E.V.S. Oliveira 275 & 25596 \\
\hline \multicolumn{10}{|l|}{ Violaceae } \\
\hline Pombalia arenaria (Ule) Paula-Souza & & $\mathrm{x}$ & & $\mathrm{x}$ & $\mathrm{Ca}$ & $\mathrm{Au}$ & Th & $\begin{array}{c}\text { E.V.S. Oliveira } \\
266 \mathrm{~A}\end{array}$ & 30577 \\
\hline Pombalia calceolaria (L.) Paula-Souza & & & $\mathrm{x}$ & & $\mathrm{Ca}$ & $\mathrm{Au}$ & Th & E.V.S. Oliveira 216 & 29035 \\
\hline \multicolumn{10}{|l|}{ Vitaceae } \\
\hline Cissus erosa Rich. & $\mathrm{x}$ & & & & $\mathrm{Ba}$ & Zo & $\mathrm{Ph}$ & E.V.S. Oliveira 300 & 30296 \\
\hline
\end{tabular}

Zoochory was the most frequent syndrome in both dune features (Fig. 3b), altough the number of species presenting different dispersal syndromes was significantly different $\left(\mathrm{x}^{2}=7.6\right.$; d.f. $=2 ; \mathrm{p}<$ 0.05 ) between the studied dune features; anemocory was proportionally more important in LD.

The proportions of life forms also recorded significant difference $\left(x^{2}=24.4 ;\right.$ d.f. $\left.=4 ; p<0.01\right)$ between the studied dune features. Phanerophytes ( $54 \%$ of the total) were the predominant life form in $\mathrm{CD}$, whereas chamaephytes and phanerophytes (approximately $30 \%$ of the total number of species, each) were proportionally more abundant in LD (Fig. 3c).

Both studied features (CD and LD) formed different groups of life form spectra when they were compared to the main Brazilian phytogeographic domains, although they showed greater resemblance to Caatinga (Fig. 4). CD was more similar to the sedimentary Caatinga when the proportion of life forms was analyzed, because both have phanerophytes and chamaephytes as their most important life forms and similar proportion of hemicryptophytes and therophytes in comparison to the total data. On the other hand, LD is more similar to the crystalline Caatinga, since both have lower proportion of phanerophytes, and higher proportion of therophytes and hemicryptophytes in comparison to the total.

The two dune features also showed significant differences in some of the analyzed soil parameters (Tab. 3): $\mathrm{pH}$, organic matter, phosphorus, sodium and base saturation index $\left(\mathrm{U}_{\text {measured }}=0\right.$ to $2 ; \mathrm{p}<$ $0.05)$. Soil analysis results per sample are available in the supplementary material $(<\mathrm{https}: / /$ doi. org $/ 10.6084 / \mathrm{m} 9$. figshare.6671792 $>$ ).

Table 2 - Richness of species and families in the dune features studied in the interior and surroundings of Biological Reserve of Santa Isabel, north coast of Sergipe state, Northeastern Brazil.

\begin{tabular}{lcccc}
\hline Feature & Species number & Family number & $\begin{array}{c}\text { Percentage of } \\
\text { exclusive species }\end{array}$ & $\begin{array}{c}\text { Distance from the } \\
\text { ocean }\end{array}$ \\
\hline Litoraneous dune & 43 & 26 & $19 \%$ & $\sim 100 \mathrm{~m}$ \\
Continental dune & 67 & 39 & $54 \%$ & $\sim 2 \mathrm{~km}$ \\
\hline
\end{tabular}



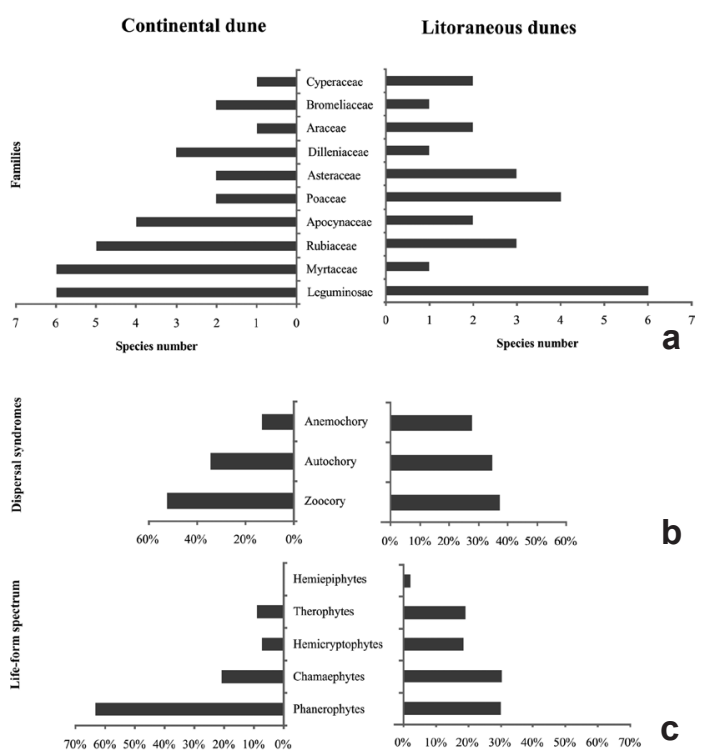

Figure 3 - The most species rich families (a), percentage of species by dispersal syndrome (b) and Raunkiaer's life forms (c) in the dune features studied in the interior and surroundings of Biological Reserve of Santa Isabel, north coast of Sergipe state, Northeastern Brazil.

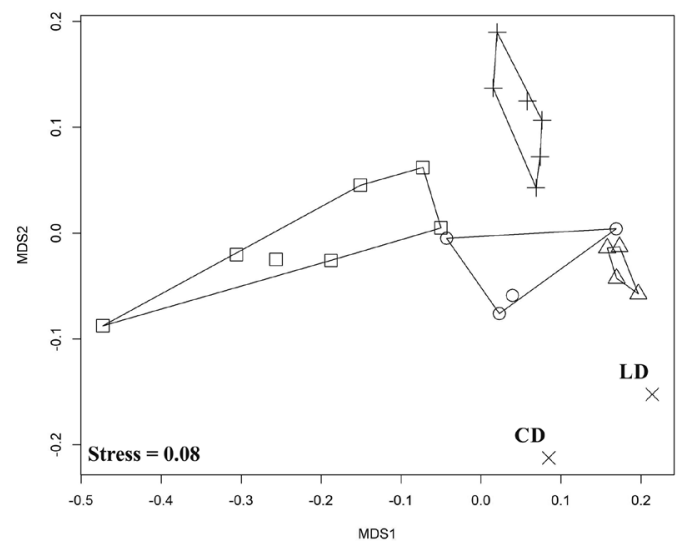

Figure 4 - Non-Metric Multidimensional Scaling (NMDS, based on the Euclidian distances) of the relation between the spectra of life forms in the main Brazilian phytogeographic domains and the dune features studied in inlands and in surrounding areas of Santa Isabel Biological Reserve, Northern coast of Sergipe state, Northeastern Brazil. Symbols representing the areas: $\square$ $=$ Ombrophilous and semideciduous Atlantic forests and the Amazonic Rainforest; + = Cerrado (Central Brazilian Savanna); $\mathrm{O}=$ Sedimentary Caatinga (Seasonally Dry Forests); $\Delta=$ Crystalline Caatinga $\times=\mathrm{CD}$ (continental dune) and LD (litoraneous dunes). Information about the areas used in the database of life forms spectra of the Brazilian phytogeographic domains were collected in Costa et al. (2016).

\section{Discussion}

Altogether, the recorded richness corresponds to $33 \%$ of the number of species found by Nascimento-Jr. (2012) in a Resting $a$ area on the Coastal Tablelands of the Northern Coast of Sergipe state. This number represents $35 \%$ of the total number of species and $55 \%$ of families already found in Santa Isabel Biological Reserve (Oliveira et al. 2015). This outcome evidences how the studied dune features hold a considerable part of the plant diversity in this protected area. We also highlight the low similarity between the two habitats studied. The Sørensen similarity between the two dune types $\left(\mathrm{S}_{\mathrm{I}}=0.36\right)$, was less than 0.50 (Felfili et al. 1992; Silva-Jr. 2001) (Tab. 2).

The proximity of litoraneous dunes (LD) to the sea when compared to continental dunes (CD) make them more prone to be influenced by the severity of sea stressing factors (salinity, wind, etc.) than CD (e.g., Cordazzo \& Seeeliger 1995; Wilson \& Sykes 1999). Assumingly, the proximity to the sea seems to be responsible for differences in the richness and floristic composition between dune features, since this pattern was also observed in other studies (e.g., Waechter 1985; Falkenberg 1999; Amaral et al. 2008). Salt spray, desiccation, wind speed and soil instability and salinity are among the main micro-environmental factors influencing plant settlement in coastal dunes (Wilson \& Sykes 1999; Maun 2009). These factors are stronger in LD due to the proximity of the sea, and to their association with lower availability of nutrition resources in the substrate (Vince \& Snow 1984; Oliveira-Filho 1993; Cordazzo \& Seeeliger 1995).

This clear micro-environmental distinction leads to zonation of species and to significant floristic richness and dissimilarity increase from the sea towards more continental areas (Andrade 1967; Waechter 1985; Oliveira-Filho 1993; Cordazzo \& Seeeliger 1995; Melo-Jr. \& Boeger 2015). Studies carried out in Paraíba (OliveiraFilho 1993; Santos et al. 2000), Sergipe (Santos et al. 2011) and Santa Catarina states (Melo-Jr. \& Boeger 2015) have shown that the structural and floristic differences among dunes on regions close to the sea within the Coastal Plain (for example, foredunes), and those dunes in further inland in this geomorphological unit (for instance, parabolic dunes) or dunes in the Coastal Tablelands can be attributed to the micro-environmental gradient. 
Table 3 - Results of the soil chemical analysis, with average and standard deviation of the samples in the dune features studied in the interior and surroundings of Biological Reserve of Santa Isabel, north coast of Sergipe state, Northeastern Brazil.

\begin{tabular}{lccc}
\hline Parameter & Unit & LD & CD \\
\hline $\mathrm{pH}^{*}$ & -- & $6.40 \pm 0.33$ & $5.91 \pm 0.28$ \\
Organic matter* & $\mathrm{g} / \mathrm{dm}^{3}$ & $3.02 \pm 1.44$ & $7.97 \pm 1.47$ \\
Aluminum (Al) & $\mathrm{cmolc} / \mathrm{dm}^{3}$ & $0.08 \pm 0.00$ & $0.10 \pm 0.01$ \\
Calcium (Ca) & $\mathrm{cmolc} / \mathrm{dm}^{3}$ & $0.24 \pm 0.20$ & $0.37 \pm 0.13$ \\
Phosphorus (P)* & $\mathrm{mg} / \mathrm{dm}^{3}$ & $4.60 \pm 2.00$ & $1.90 \pm 0.45$ \\
Magnesium (Mg) & $\mathrm{cmolc} / \mathrm{dm}^{3}$ & $0.77 \pm 0.24$ & $0.58 \pm 0.15$ \\
Potassium (K) & $\mathrm{mg} / \mathrm{dm}^{3}$ & $17.60 \pm 7.69$ & $8.00 \pm 0.90$ \\
Sodium (Na)* & $\mathrm{mg} / \mathrm{dm}^{3}$ & $6.20 \pm 2.73$ & $3.00 \pm 0.75$ \\
Exchangeable bases & $\mathrm{cmolc} / \mathrm{dm}_{3}$ & $1.09 \pm 0.37$ & $0.88 \pm 0.22$ \\
Cation exchange capacity & $\mathrm{cmolc} / \mathrm{dm}_{3}$ & $1.46 \pm 0.46$ & $1.42 \pm 0.60$ \\
Base saturation index* & $\%$ & $73 \pm 11$ & $53 \pm 8$ \\
\hline
\end{tabular}

$*=$ statistically significant difference.

However, the role played by historical processes such as the geological history of the studied features (Barthlott et al. 2005) can not be ruled out at the time to explain the recorded results. Although both dune features were formed in the Quaternary period, they differ in Epoch formation: $\mathrm{CD}$ was formed in the Pleistocene and LD in the Holocene (Salgado-Labouriau 1994; Alves 2010). At least six major events of Quaternary sea level variation were observed in the Northern coast of Sergipe state: three transgression events and three regression ones (Bittencourt et al. 1983; Suguio $\&$ Tesller 1984).

Assumingly, dunes in CD were formed prior to the Cananéia Transgression, in the subsequent regression to Mais Antiga Transgression (older than 120,000 years B.P.), which was the first of the three greatest transgressive Quaternary episodes in the Northern coast of Sergipe state. On the other hand, dunes in LD are more recent than 5,100 years B.P., because their formation during the subsequent regression to Santos Transgression, which was the last of the six main sea level variation events in the Quaternary in the North coast of Sergipe State (Bittencourt et al. 1983; Suguio \& Tesller 1984). Longer existence times such as that of CD allows more complete colonization than more recent areas (Currie 1991; Diniz-Filho \& Bini 2011).

The Leguminosae stands out as one of the richest family in surveys conducted in coastal dunes in many Brazilian states, namely: Santa Catarina (Ribeiro \& Melo-Jr. 2016; Melo-Jr. et al. 2017), Bahia (Brito et al. 1993; Viana et al. 2006), Alagoas (Almeida-Jr. et al. 2016), Pernambuco (Leite \& Andrade 2004), Rio Grande do Norte (Freire 1990), Maranhão (Araujo et al. 2016) and Pará (Santos \& Rosário 1988). Furthermore, this family has high diversity in the dunes and Coastal Tablelands areas of the Northern coast of Sergipe state (Silva 2014). Leguminosae species are very efficient in colonizing sterile environments, such as sand, they prefer dry and warm areas (Carvalho \& Oliveira-Filho 1993; Stehmann et al. 2009), fact that may explain the high importance of this family to both analyzed features.

Asteraceae and Poaceae were among the four richest families in species number in LD, besides being the most important in dunes located in the Coastal Plains of Rio Grande do Sul (Palma \& Jarenkow 2008), Rio de Janeiro (Castelo \& Braga 2017), and Sergipe states (Santos et al. 2011); these families are mostly composed of herbaceous species that colonize open areas. The influence of the wind is of fundamental importance to their pollination and dispersal (Carvalho \& OliveiraFilho 1993; Palma \& Jarenkow 2008; Souza \& Lorenzi 2012). This process can explain the highest distribution of these families in LD, whose environmental characteristics (e.g, higher wind intensity) may be more favorable than CD. 
Myrtaceae, which is one of the richest families in $\mathrm{CD}$, presents high diversity in coastal forests (e.g., Pereira \& Assis 2000; Lourenço \& Barbosa 2012). Their floristic importance is greater in studies carried out in coastal scrubs (arbustais or fruticetos in Portuguese) and Restinga forests in Northeast Brazil: Rio Grande do Norte (AlmeidaJr. \& Zickel 2012), Pernambuco (Sacramento et al. 2007) and Alagoas states (Medeiros et al. 2010); in Southeast Brazil: Espírito Santo (Assis et al. 2004), Rio de Janeiro (Sá \& Araújo 2009) and São Paulo states (Guedes et al. 2006); and in South Brazil: Rio Grande do Sul state (Scherer et al. 2005; Santos et al. 2012). Mytaceae diversity in coastal forests (Menezes-Silva 1998; Souza \& Lorenzi 2012) can justify their importance in CD.

Significant differences in the proportion of dispersal syndromes and in Raunkiaer's life forms between LD and CD were attributed to plant adaptive responses to micro-environmental variations between these features. Therefore, these results may be attributed to selective pressures acting on these environments, mainly in LD. A similar pattern was observed in two Restinga formations in Santa Catarina state, whose differences in vegetation were attributed to the selective pressure exerted by environmental factors (Melo-Jr. et al. 2017). Evidences indicate pressure of environmental filters on the region near the sea, which leads to convergent morphological adjustments between plants (Melo-Jr. \& Boeger 2017).

Zoochory was the most important dispersal syndrome in both analyzed features, it is one of the most frequent syndromes in coastal dunes, mainly in leeward regions (Marques \& Oliveira 2005). Restinga vegetation is structurally more developed in these environments, since it forms scrubs (fruticetos) and coastal forests (OliveiraFilho \& Carvalho 1993; Oliveira \& Landim 2014). Anemochory, which is one of the most important syndromes in LD, may be the most efficient along the coast, since most species distributed in dune windwards are wind dispersed (Maun 2009).

The CD distribution in areas more protected from the windy can benefit other syndromes to the detriment of anemochory. The same process can also happen in the LD leeward, fact that justifies the high zoochory proportion in this feature. LD has been colonized by species from adjacent open fields where autochoric and anemochoric species are usually prevalent (Marques \& Oliveira 2005). Thus, anemochory should be more advantageous for LD plants than zoochory, which is more advantageous in CD.

Phanerophytes had curved stem and treetops following the prevailing direction of the winds in LD. This outcome is known as wind pruning or anamorphic aspect (Waechter 1985; AlmeidaJr. \& Zickel 2009; Castelo \& Braga 2017). Phanerophyte, which is the first most expressive life form in $\mathrm{CD}$ and $\mathrm{LD}$, was also important for Restinga areas in Maranhão (Serra et al. 2016), Rio Grande do Norte (Almeida-Jr. et al. 2006; Almeida-Jr. \& Zickel 2009), Pernambuco (Almeida Jr. et al. 2007, 2009), Alagoas (AlmeidaJr. et al. 2016) and Bahia states (Gomes \& Guedes 2014).

Hemicryptophytes and chamaephytes can be branched or clotted to assure protection to the gemstones subjected to stressful environmental conditions such as salt spraying (Begon et al. 2006; Martins \& Batalha 2011). Such characteristics may suggest more evolutionary advantages to these life forms than to phanerophytes in LD. This outcome corroborates the higher frequency of these life-forms in studies carried out in beach areas (Almeida-Jr. \& Zickel 2009; Araujo et al. 2016).

The ordination analysis revealed that $\mathrm{CD}$ and LD present life forms spectra different from other areas within the main phytogeographic domains of Brazil. Periods of low rainfall in coastal dunes, low water retention by the substrate, high sand temperature and high evapotranspiration rates, force plants in this environment to face water deficit (Cordazzo et al. 2006). This scenario may justify the high proportion of hemicryptophytes and therophytes (Martins \& Batalha 2011) in comparison to the total life form spectra in LD, as observed for crystalline Caatinga (Costa et al. 2016). On the other hand the stress factors causing water deficit in $\mathrm{CD}$, which is farther from the sea, are less intense (Cordazzo et al. 2006), fact that explains why its life-form spectrum is more similar to the sedimentary Caatinga rather than having potentially more water available in the soil than the crystalline Caatinga (Costa et al. 2016).

Soil chemical characteristics are other factors capable of setting the differences in the distribution, richness, floristic composition and abundance of Restinga vegetation (AlmeidaJr. et al. 2011, 2016; Santos-Filho et al. 2013; Magnago et al. 2012). Some of the soil parameters responsible for these differences are $\mathrm{pH}$, organic matter, $\mathrm{P}, \mathrm{Na}$ and base saturation index (Santos 
et al. 2000; Almeida-Jr. et al. 2009; Magnago et al. 2010, 2012; Santos-Filho et al. 2013). These parameters differed between the studied dune features.

Organic matter and sodium are some of the main soil attributes responsible for structural differences and floristic composition in Restinga areas among the aforementioned ones. They favor or limit the development of these areas and, consequently, set the phytophysiognomic type (Almeida-Jr. et al. 2009; Magnago et al. 2010, 2012; Santos-Filho et al. 2013; Melo-Jr. \& Boeger 2015). These two attributes are amid the ones mostly limiting species richness in dune areas at the beginning of the successional process (Santos et al. 2000).

The presence of organic matter modifies the environment by facilitating nutrient absorption and by increasing particle aggregation and moisture retention in the substrate (Silva \& Sommer 1984). Most plants are not tolerant to sodium; therefore, high concentrations of it can be very harmful to some species (Epstein \& Bloom 2006). The highest amount of organic matter and the lowest sodium content in CD soils may have contributed to its greater richness and subsidized the observed floristic composition differences between the two studied dune features.

The lack of information about the optimal ranges of soil chemical attribute values for Restinga plants impairs the development of more robust explanations about the observed differences. However, data presented in the scientific literature substantiate the conclusion that organic matter and $\mathrm{Na}$ are somehow related to the observed differences in the vegetation of both dune features.

\section{Supplementary material}

All figures, the list of species and the raw data (e.g., soil data) used to support our analysis are available for download at $<$ https://doi.org/10.6084/ m9.figshare.6671792>.

\section{Acknowledgements}

The authors are grateful to the Conselho Nacional de Desenvolvimento Científico e Tecnológico $(\mathrm{CNPq})$ for the scientific initiation scholarship granted to the first author; to the ITPS (Instituto Tecnológico e de Pesquisas do Estado de Sergipe) by soil analysis; to the UFS (Universidade Federal de Sergipe) for providing transport and laboratory facilities; to the ASE Herbarium staff for their help; to the Instituto Chico Mendes de Conservação da Biodiversidade (ICMBio) by the collection authorization number 41647.

\section{References}

Almeida-Jr. EB \& Zickel CS (2009) Fisionomia psamófila-reptante: riqueza e composição de espécies na praia da Pipa, Rio Grande do Norte, Brasil. Pesquisas, Botânica 60: 289-299.

Almeida-Jr. EB \& Zickel CS (2012) Análise fitossociológica do estrato arbustivo-arbóreo de uma floresta de restinga no Rio Grande do Norte. Revista Brasileira de Ciências Agrárias 7: 286-291.

Almeida-Jr. EB, Zickel CS \& Pimentel RMM (2006) Caracterização e espectro biológico da vegetação do litoral arenoso do Rio Grande do Norte. Revista de Geografia 23: 45-58.

Almeida-Jr. EB, Pimentel RMM \& Zickel CS (2007) Flora e formas de vida em uma área de restinga no litoral Norte de Pernambuco, Brasil. Revista de Geografia 24: 19-34.

Almeida-Jr. EB, Olivo MA, Araujo EL \& Zickel CS (2009) Caracterização da vegetação de restinga da RPPN de Maracaípe, PE, Brasil, com base na fisionomia, flora, nutrientes do solo e lençol freático. Acta Botanica Brasilica 23: 36-48.

Almeida-Jr. EB, Machado MA, Medeiros DPW, Pinheiro TS \& Zickel CS (2016) Florística de uma área de vegetação de influência marinha no litoral sul de Alagoas, Brasil. Revista Brasileira de Geografia Física 9: 1400-1409.

Almeida-Jr. EB, Santos-Filho FS, Araújo EL \& Zickel CS (2011) Structural characterization of the woody plants in restinga of Brazil. Journal of Ecology and the Natural Environment 3: 95-103.

Alvares CA, Stape JL, Sentelhas PC, Gonçalves JLM \& Sparovek G (2013) Köppen's climate classification map for Brazil. Meteorologische Zeitschrift 22: 711-728.

Alves NMS (2010)Análise geoambiental e socioeconômica dos municípios costeiros do litoral norte do estado de Sergipe - diagnóstico como subsidio ao ordenamento e gestão do território. Tese de Doutorado. Universidade Federal de Sergipe, São Cristóvão. 348p.

Amaral DD, Prost MT, Bastos MNC, Neto SVC \& Santos JUM (2008) Restingas do litoral amazônico, estados do Pará e Amapá, Brasil. Boletim do Museu Paraense Emílio Goeldi 3: 35-67.

Andrade MAB (1967) Contribuição ao conhecimento da ecologia das plantas das dunas do litoral do estado de São Paulo. Tese de Doutorado. Universidade de São Paulo, São Paulo. 167p.

APG IV - Angiosperm Phylogeny Group (2016) An update of the Angiosperm Phylogeny Group classification for the orders and families of flowering plants: APG IV. Botanical Journal of the Linnean Society $181: 1-20$. 
Araujo ACM, Silva ANF \& Almeida-Jr. EB (2016) Caracterização estrutural e status de conservação do estrato herbáceo de dunas da Praia de São Marcos, Maranhão, Brasil. Acta Amazonica 46: 247-258.

Assis AM, Thomaz LD \& Pereira OJ (2004) Florística de um trecho de floresta de restinga no município de Guarapari, Espírito Santo, Brasil. Acta Botanica Brasilica 18: 191-201.

Barroso GM, Morin ML, Peixoto AL \& Ichaso CLF (2004) Frutos e sementes. Editora UFV, Viçosa. $443 p$.

Barthlott W, Mutke J, Rafiqpoor D, Kier G \& Kreft H (2005) Global centers of vascular plant diversity. Nova Acta Leopoldina 92: 61-83.

Begon M, Townsend CR \& Harper JL (2006) Ecology: from individuals to ecosystems. $4^{\text {th }}$ ed. Blackwell Publishing, Oxford. 752p.

BFG - The Brazil Flora Group (2018) Brazilian Flora 2020: innovation and collaboration to meet Target 1 of the Global Strategy for Plant Conservation (GSPC). Rodriguésia 69: 1513-1527.

Bittencourt ACSP, Marti L, Dominguez JML \& Ferreira YA (1983) Evolução paleogeográfica quaternária da costa do estado de Sergipe e da costa sul do estado de Alagoas. Revista Brasileira de Geociências 13: 93-97.

Brasil (1988) Decreto $n^{\circ} 96.999$ de 20 de outubro de 1988. Cria, no litoral do estado de Sergipe, a Reserva Biológica de Santa Isabel e dá outras providências. Available at <http://www.planalto.gov.br/ccivil_03/ decreto/1980-1989/D96999.htm>. Access on 17 November 2017.

Brito CI, Queiroz LP, Guedes MLS, Oliveira NC \& Silva LB (1993) Flora fanerogâmica das dunas e lagoas do Abaeté, Salvador, Bahia. Sitientibus 11: 31-46.

Carvalho DA \& Oliveira-Filho AT (1993) Avaliação da recomposição da cobertura vegetal de dunas de rejeito de mineração, em Mataracá/PB. Acta Botanica Brasilica 7: 107-117.

Castelo AJ \& Braga JMA (2017) Checklist of sand dune vegetation on the tropical southeastern Brazil coast. Check List 13: 1-12.

Cerqueira R (2000) Biogeografia das restingas. In: Esteves FA \& Lacerda LD (eds.) Ecologia de restingas e lagoas costeiras. NUPEM/UFRJ, Macaé. Pp. 65-75.

Cordazzo CV \& Seeliger U (1995) Guia ilustrado da vegetação costeira no extremo sul do Brasil. $2^{\text {nd }} \mathrm{ed}$. Editora da FURG, Rio Grande. 275p.

Cordazzo CV, Paiva JB \& Seeliger U (2006) Guia ilustrado: plantas das dunas da costa sudoeste Atlântica. USEB, Pelotas. 107p.

Costa ACM, Moro MF \& Martins FR (2016) Raunkiaerian life-forms in the Atlantic forest and comparisons of life-form spectra among Brazilian main biomes. Brazilian Journal of Botany 39: 833-844.

Currie D (1991) Energy and large-scale patterns of animal and plant-species richness. The American Naturalist 137: 27-49.
Diniz-Filho JAF \& Bini LM (2011) Geographical patterns in biodiversity: towards an integration of concepts and methods from genes to species diversity. Natureza \& Conservação 9: 179-187.

EMBRAPA-Empresa Brasileira de Pesquisa Agropecuária (2006) Sistema Brasileiro de Classificação de Solos. EMBRAPA-SPI, Rio de Janeiro. 306p.

EMBRAPA - Empresa Brasileira de Pesquisa Agropecuária / SUDENE - Superintendência do Desenvolvimento do Nordeste (1975) Levantamento exploratório - reconhecimento de solos do estado de Sergipe. EMBRAPA/SUDENE, Recife. 545p.

Epstein E \& Bloom JA (2006) Nutrição mineral de plantas. Editora Planta, Londrina. 404p.

Falkenberg DB (1999) Aspectos da flora e da vegetação secundária da restinga de Santa Catarina, Sul do Brasil. Insula 28: 1-30.

Felfili JM, Silva-Jr. MC, Rezende AV, Machado JWB, Walter BMT, Silva PEN \& Hay JD (1992) Análise comparativa da florística e fitossociologia da vegetação arbórea do Cerrado Sensu Stricto na chapada Pratinha, DF-Brasil. Acta Botanica Brasilica 6: 1992.

Filgueiras TS, Nogueira PE, Brochado AL \& Guala GF (1994) Caminhamento: um método expedito para levantamentos florísticos qualitativos. Cadernos de Geociências 12: 39-43.

Fontes AL (1985) Geomorfologia da área de Pirambu e adjacência - Sergipe. Dissertação de Mestrado. Universidade Federal da Bahia, Salvador. 152p.

Fraga RZ (2010) Análise ecodinâmica da Reserva Biológica de Santa Isabel. Agirás 2: 61-88.

Freire MSB (1990) Levantamento florístico do Parque Estadual das Dunas de Natal. Acta Botanica Brasilica 4: 41-59.

Gomes FS \& Guedes MLS (2014) Flora vascular e formas de vida das formações de restinga do litoral norte da Bahia, Brasil. Acta Biológica Catarinense 1: 22-43.

Guara M, Laguna E \& Currás R (1992) Espectro de las formas biológicas de Raunkiaer en lãs dunas de Guardamar Del Segura y Elche. Cuadernos de Geografía 52: 149-156.

Guedes D, Barbosa LM \& Martins SE (2006) Composição florística e estrutura fitossociológica de dois fragmentos de floresta de restinga no município de Bertioga, SP, Brasil. Acta Botanica Brasilica 20: 299-311.

Guerra AJT \& Cunha SB (2009) Geomorfologia: uma atualização de bases e conceitos. Bertrand Brasil, Rio de Janeiro. 474p.

Guerra AT \& Guerra ATJ (2008) Novo dicionário geológico-geomorfológico. Bertrand Brasil, Rio de Janeiro. 652p.

Gurevitch J, Scheiner SM \& Fox GA (2009) Ecologia Vegetal. Artmed, Porto Alegre. 592p.

Hammer Ø, Harper DAT \& Ryan PD (2013) PAST Palaeontological statistics. Available at $<\mathrm{http}$ ://folk. uio.no/ohammer/past/>. Access on 10 June 2017. 
Henriques MV \& Carlos-Neto (2002) Caracterização geo-ecológica dos sistemas de cordões dunares de Estremadura (Nazaré, S. Martinho do Porto, Peniche-Baleal). Finisterra 74: 5-31.

I3N Brasil / Instituto Hórus (2018) Base de dados nacional de espécies exóticas invasoras. Available at $<$ http://i3n.institutohorus.org.br/www $>$. Access on 15 November 2018.

ICMBio - Instituto Chico Mendes de Conservação da Biodiversidade (2017) Limites das unidades de conservação federais. Available at $<$ http://www. icmbio.gov.br/portal/geoprocessamento 1/51menu-servicos/4004-downloads-mapa-tematicoe-dados-geoestatisticos-das-uc-s. $>$. Access on 10 December 2017.

Joly AB (1970) Conheça a vegetação brasileira. Edusp, São Paulo. 181p.

Leite AVL \& Andrade LHC (2004) Riqueza de espécies e composição florística em um ambiente de duna após 50 anos de pressão antrópica: um estudo na praia de Boa Viagem, Recife, PE, Brasil. Biotemas 17: 29-46.

Lorenzi H, Bacher L, Lacerda M \& Sartori S (2006) Frutas brasileiras e exóticas cultivadas: de consumo in natura. Instituto Plantarum, Nova Odessa. 672p.

Lourenço ARL \& Barbosa MRV (2012) Myrtaceae in the restinga vegetation at the northern limit of the Atlantic coastal forest, Brazil. Rodriguésia 63: 373-393.

Magnago LFS, Martins SM, Schaefer CEGR \& Neri AV (2010) Gradiente fitofisionômico-edáfico em formações florestais de Restinga no sudeste do Brasil. Acta Botanica Brasilica 24: 734-746.

Magnago LFS, Martins SV, Schaefer CEGR \& Neri AV (2012) Restinga forests of the Brazilian coast: richness and abundance of tree species on different soils. Anais da Academia Brasileira de Ciências 84: 807-822.

Marques MCM \& Oliveira PEAM (2005) Características reprodutivas das espécies vegetais da planície litorânea. In: Marques MCM \& Britez RM (eds.) História natural e conservação da Ilha do Mel. Editora UFPR, Curitiba. Pp. 169-189.

Martin JS, Ramirez C \& Martin CS (1992) La flora de las dunas chilenas y sus adaptaciones morfológicas. Bosque 13: 29-39.

Martins FR \& Batalha MA (2011) Formas de vida, espectro biológico de Raunkiaer e fisionomia da vegetação. In: Felfili JM, Eisenlohr PV, Melo MMRF, Andrade LA \& Meira-Neto JAA(eds.) Fitossociologia no Brasil: métodos e estudos de caso. Editora UFV, Viçosa. Pp. 44-85.

Martins-da-Silva RCV, Silva ASL, Fernandes MM \& Margalho LF (2014) Noções morfológicas e taxonômicas para identificação botânica. Embrapa, Brasília. 111p.

Maun MA (2009) The biology of coastal sand dunes. Oxford University, New York. 280p.
Medeiros DP, Santos-Filho FS, Almeida-Jr. EB, Pimentel RMM \& Zickel CS (2010) Estrutura do componente lenhoso de uma Restinga no litoral sul de Alagoas, Nordeste, Brasil. Revista Brasileira de Geografia Física 3: 146-150.

Melo-Jr. JCF \& Boeger MRT (2015) Riqueza, estrutura e interações edáficas em um gradiente de restinga do Parque Estadual do Acaraí, estado de Santa Catarina, Brasil. Hoehnea 42: 207-232.

Melo-Jr. JCF \& Boeger MRT (2017) Functional traits of dominant plant species of the Brazilian sandy coastal plain. International Journal of Current Research 9: 45585-45593.

Melo-Jr. JCF, Ferrari A, Gern AF, Daniel A, Maia AC, Hartelt BT, Possamai BT, Vanzuita CA, Dumke D, Silva D, Tussolini EGR, Hafemann F, Silva GR, Oliveira GB, Jantsch G, Batista JM, Ferreira J, Cruzzeta KM, Teixeira LRRV, Burmester LP, Santos MC, Sabino PM, Valdez PE, Jasper RB, Pinto RF, Oliveira S \& SeifertNeto VR (2017) Fitossociologia comparada da comunidade herbáceo-subarbustiva de restinga em duas geoformações de dunas na planície costeira de Santa Catarina. Acta Biológica Catarinense 4: 5-15.

Menezes-Silva S (1998) As formações vegetais da planície litorânea da Ilha do Mel, Paraná, Brasil: composição florística e principais características estruturais. Tese de Doutorado. Universidade Estadual de Campinas, Campinas. 55p.

Mori SA, Silva LAM, Lisboa G \& Coradin L (1989) Manual de manejo do herbário fanerogâmico. Centro de Pesquisas do Cacau, Ilhéus. 104p.

Moro MF, Macedo MB, Moura-Fé MM, Castro ASF \& Costa RC (2015) Vegetação, unidades fitoecológicas e diversidade paisagística do estado do Ceará. Rodriguésia 66: 717-743.

Mueller-Dombois D \& Ellenberg H (1974) Aims and methods of vegetation ecology. John Wiley \& Sons, New York. 547p.

Nascimento-Jr. JE (2012) Flora eletrônica de um trecho do litoral norte de Sergipe, Brasil. Dissertação de Mestrado. Universidade Estadual de Campinas, Campinas. 549p.

Oksanen J, Blanchet FG, Friendly M, Kindt R, Legendre P, McGlinn D, Minchin PR, O'Hara RB, Simpson GL, Solymos P, Stevens MHM, Szoecs E \& Wagner H (2018) Vegan: community ecology package. R package version 2.5-1. Available at $<$ https://CRAN.R-project.org/package $=$ vegan $>$. Access on 10 June 2018.

Oliveira EVS, Lima JF, Silva TC \& Landim MF (2014) Checklist of the flora of the Restingas of Sergipe state, Northeast Brazil. Check list 10: 529-549.

Oliveira EVS \& Landim MF (2014) Caracterização fitofisionômica das restingas da Reserva Biológica de Santa Isabel, litoral Norte de Sergipe. Scientia Plena 10: 1-10. 
Oliveira EVS, Ferreira-Sobrinho ES \& Landim MF (2015) Flora from the restingas of Santa Isabel Biological Reserve, northern coast of Sergipe state, Brazil. Check List 11: 1-10.

Oliveira EVS \& Landim MF (2016) Flora das Restingas de Sergipe: padrões de distribuição espacial e status de conservação de suas espécies. Natureza Online 14: 23-31.

Oliveira-Filho AT (1993) Gradient analysis of an area of coastal vegetation in the state of Paraiba. Northeastern Brazil. Edinburgh Journal of Botany 50: 217-236.

Oliveira-Filho AT \& Carvalho DA (1993) Florística e fisionomia da vegetação no extremo norte do litoral da Paraíba. Revista Brasileira de Botânica 16: 115-130.

Palma CB \& Jarenkow JA (2008) Estrutura de uma formação herbácea de dunas frontais no litoral norte do RS, Brasil. Biociências 16: 114-124.

Pereira OJ \& Assis AM (2000) Florística da restinga de Camburi, Vitória, ES. Acta Botanica Brasilica 14: 99-111.

Pergentino TC \& Landim MF (2014) Restingas de Sergipe: contribuição ao conhecimento da sua composição florística e análise sobre o status de conservação atual. In: Landim MF \& Guimarães CP (eds.) Ecologia, uso potencial e conservação de ecossistemas costeiros sergipanos. Editora UFS, São Cristovão. Pp. 103-128.

Pijl LVD (1982) Principles of dispersal in higher plants. Springer-Verlag, Berlim. 214p.

Prata APN, Amaral MCE, Farias MCV \& Alves MV (2013) Flora de Sergipe (Vol. 1). Gráfica e Editora Triunfo, Aracaju. 592p.

Prata APN, Farias MCV \& Landim MF (2015) Flora de Sergipe (Vol. 2). Editora Criação, Aracaju. 301p.

Ribeiro PY \& Melo-Jr. JCF (2016) Richness and community structure of sand dunes (restinga) in Santa Catarina: subsidies for ecological restoration. Acta Biológica Catarinense 3: 25-35.

R Development Core Team (2013) R: a language and environment for statistical computing. R Foundation for Statistical Computing, Vienna. Available at $<$ https://www.r-project.org/>. Access on 10 June 2018.

Rizzini AT (1997) Tratado de fitogeografia do Brasil: aspectos ecológicos, sociológicos, e florísticos. Âmbito Cultural, Rio de Janeiro. 747p.

Sá CFC \& Araujo DSD (2009) Estrutura e florística de uma floresta de restinga em Ipitangas, Saquarema, Rio de Janeiro, Brasil. Rodriguésia 60: 147-170.

Sacramento ACS, Almeida-Jr. EB \& Zickel CS (2007) Aspectos florísticos da vegetação de restinga no litoral de Pernambuco. Revista Árvore 31: 1121-1130.

Salgado-Labouriau ML (1994) História ecológica da terra. Edgard Blucher, São Paulo. 320p.

Santos JUM \& Rosário CS (1988) Levantamento da vegetação fixadora de dunas de Algodoal-PA.
Boletim do Museu Paraense Emílio Goeldi 4: 133-151.

Santos M, Rosado SCS, Oliveira-Filho AT \& Carvalho D (2000) Correlações entre variáveis do solo e espécies herbáceo-arbustivas de dunas em revegetação no litoral norte da Paraíba. Cerne 6: 19-29.

Santos SSC, Reis VS, Angelo-Furlan S \& Melo \& Souza R (2011) Biodiversidade e potencial fitoindicador da vegetação de dunas costeiras da Barra dos Coqueiros, Sergipe, Brasil. Revista IberoAmericana de Ciências Ambientais 2: 5-20.

Santos R, Silva CR, Pacheco D, Martins R \& CitadiniZanette V (2012) Florística e estrutura do componente arbustivo-arbóreo de mata de restinga arenosa no Parque Estadual de Itapeva, Rio Grande do Sul. Revista Árvore 36: 1047-1059.

Santos EAP, Landim MF, Oliveira EVS \& Silva ACCD (2017) Conservação da zona costeira e áreas protegidas: a Reserva Biológica de Santa Isabel (Sergipe) como estudo de caso. Natureza online 15: 41-57.

Santos-Filho FS, Almeida-Jr. RB \& Zickel CS (2013) Do edaphic aspects alter vegetation structures in the Brazilian restinga? Acta Botanica Brasilica 27: 613-623.

Scherer A, Maraschin-Silva F \& Baptista LRM (2005) Florística e estrutura do componente arbóreo de matas de Restinga arenosa no Parque Estadual de Itapuã, RS, Brasil. Acta Botanica Brasilica 19: 717-726.

SEMARH / SRH-SE (2013) Atlas digital sobre recursos hídricos de Sergipe. Aracaju. Available at $<$ https:// www.semarh.se.gov.br/recursoshidricos/?page $\mathrm{id}=486>$. Access on 10 December 2017.

SEPLAG-SE (2011) Sergipe em dados. Supes, Aracaju. $101 \mathrm{p}$.

SEPLANTEC-SE (2003) Base cartográfica dos municípios litorâneos de Sergipe. Fotografias aéreas (cobertura do município de Pirambu). Escala 1:25.000. Aracaju. 1 CD-ROM

Serra FCV, Lima PB \& Almeida-Jr. EB (2016) Species richness in restinga vegetation on the eastern Maranhão state, Northeastern Brazil. Acta Amazonica 46: 271-280.

Siegel S (1977) Estatística não-paramétrica (para as ciências do comportamento). McGraw-Hill do Brasil, São Paulo. 350p.

Silva FC (1999) Manual de análises químicas de solos, plantas e fertilizantes. Embrapa Solos, Brasília. $627 \mathrm{p}$.

Silva JG \& Somner GV (1984) A vegetação da restinga na Barra de Maricá, RJ. In: Lacerda LD, Araújo DSD, Cerqueira R \& Turcq B (eds.) Restingas: origem, estrutura e processos. CEUFF, Niterói. Pp. 217-225.

Silva TC (2014) Diversidade de Leguminosae Juss. na Restinga e nos Tabuleiros de Pirambu, Sergipe, Brasil. Dissertação de Mestrado. Universidade Estadual de Campinas, Campinas. 158p. 
Silva-Jr. MC (2001) Comparação entre matas de galeria no distrito federal e a efetividade do código florestal na proteção de sua diversidade arbórea. Acta Botanica Brasilica 15: 139-146.

Souza VC \& Lorenzi H (2012) Botânica Sistemática: guia ilustrado para identificação das famílias de Fanerógamas nativas e exóticas no Brasil, baseado em APG III. Instituto Plantarum, Nova Odessa. $768 \mathrm{p}$.

Stehmann JR, Forzza RC, Sobral M \& Kamino LHY (2009) Gimnospermas e Angiospermas. In: Stehmann JR, Forzza RC, Salino A, Sobral M, Costa DP \& Kamino LHY (eds.) Plantas da Floresta Atlântica. Instituto de Pesquisas do Jardim Botânico do Rio de Janeiro, Rio de Janeiro. Pp. 372-403.

Suguio K \& Tessler MG (1984) Planícies de cordões litorâneos quaternários do Brasil: origem e nomenclatura. In: Lacerda LD, Araujo DSD, Cerqueira R \& Turcq B (eds.) Restingas: origem, estrutura e processos. Editora UFF, Rio de Janeiro. Pp. 15-25.
Viana BF, Silva FO \& Kleinert AMP (2006) A flora apícola de uma área restrita de dunas litorâneas, Abaeté, Salvador, Bahia. Revista Brasileira de Botanica 29: 13-25.

Villwock JE, Lessa GC, Suguio K, Ângulo RJ \& Dillenburg DR (2005) Geologia e geomorfologia de regiões costeiras. In: Souza CRG, Suguio K, Oliveira AMS \& Oliveira PE (eds.) Quaternário do Brasil. Holos, Ribeirão Preto. Pp. 94-107.

Vince SW \& Snow AA (1984) Plant zonation in an Alaskan salt marsh: I. distribution, abundance and environmental factors. Journal of Ecology 72 : 651-667.

Waechter JL (1985) Aspectos ecológicos da vegetação de restinga no Rio Grande do Sul, Brasil. Comunicações do museu de ciências PUC-RS 33: 49-68.

Wilson JB \& Sykes MT (1999) Is zonation on coastal sand dunes determined primarily by sand burial or salt spray? A test in New Zealand dunes. Ecology letters 2: 233-236. 